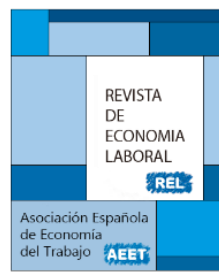

\title{
LA RIGIDEZ DEL COSTE LABORAL Y EL CRECIMIENTO DEL EMPLEO EN ESPAÑA ${ }^{1}$
}

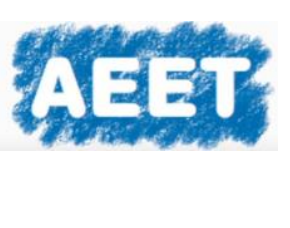

\author{
Marta Martínez-Matute ${ }^{2}$ \\ Banco de España y Syracuse University
}

Recibido Septiembre 2016; Aceptado Noviembre 2016

\section{Resumen}

Este artículo pretende estudiar el efecto de la negociación colectiva sobre el empleo a través de una vía indirecta: la rigidez que provoca el mecanismo para fijar los salarios. La negociación colectiva es una de las causas principales de la rigidez salarial del mercado laboral en España. Se propone la construcción de un "indice de rigidez del coste laboral" que refleja la falta de ajuste entre las diferencias en la productividad y las diferencias del coste laboral, el cual se emplea posteriormente para estimar un modelo que explique las variaciones del empleo durante 1995-2014. Los resultados indican que la rigidez del coste laboral afecta de forma negativa y significativa al crecimiento del empleo.

Palabras clave: Negociación colectiva, Sindicatos, Rigidez del coste laboral, Crecimiento del empleo, Empresas españolas.

Clasificación JEL: J31, J51, J52

\section{Abstract}

This paper seeks to explain the effect of collective bargaining on the employment through and indirect way: the rigidity of the wage-setting mechanism. Collective bargaining is one of the main causes of the rigidity of the Spanish labor market. We propose an "index of labor cost rigidity", which reflects the lack of adjustment of the differences in productivity and in wages, and we use it to estimate the employment growth during 1995-2014. The results indicate that the rigidity of the labor costs affects negative and significantly to the employment growth.

Key words: Collective bargaining, Unions, Rigidity of labor cost, Employment growth, Spanish firms.

JEL Classification: J31, J51, J52

1 La autora agradece a José Miguel Sánchez Molinero la realización de este artículo. Asimismo, agradece los comentarios de Ángel Martín, Alfonso Moral y Carlos Pérez, así como los comentarios hechos por el evaluador anónimo y los recibidos en las X Jornadas de Economía Laboral, en los XVI Encuentros de Economía Aplicada y en los seminarios de investigación de la Universidad Autónoma de Madrid y en la Universidad de Oviedo. Las opiniones y análisis expresados en este artículo son responsabilidad exclusiva de la autora y, por tanto, no comprometen al Banco de España ni a Syracuse University.

${ }^{2}$ Autora para correspondencia: marta.martinez@bde.es

(C) Revista de Economía Laboral 


\section{Introducción}

En España la negociación colectiva está escasamente articulada entre los distintos ámbitos funcionales y regionales. La falta de flexibilidad del mecanismo mediante el que se fijan las tarifas salariales incide de manera decisiva en la vía de ajuste del empleo ante cambios en la coyuntura económica. Existe ya una amplia literatura previa en la que se analizan el efecto que tienen los distintos tipos de convenios colectivos en el empleo y el desempleo regional y sectorial (Lorences et al., 1995; Pérez Infante, 2003; Bande et al., 2010).

La cobertura de la negociación colectiva en España es muy alta (entre el $80 \%$ y el $90 \%$ de los trabajadores tienen un convenio colectivo que les cubre), un dato que contrasta claramente con el nivel de afiliación sindical (de en torno al 15\%), y que es uno de los más bajos de Europa. Además, su grado de coordinación y de centralización intermedia, donde predominan los convenios colectivos sectoriales y provinciales, es el menos propicio para adaptar los salarios la situación económica de las empresas y el que más distorsiona el funcionamiento de la economía en general (Bentolila y Jimeno, 2002; Palacio y Simón, 2002). De este modo, las tarifas salariales no reflejan bien las diferencias de cualificación y productividad y no permiten adaptar los costes laborales de una empresa a su situación específica. Si los salarios se fijan con independencia de los cambios en la productividad, el empleo puede verse afectado (Bande et al., 2007). Además, Bentolila et al. (2010) mencionan varios factores en los que la estructura de la negociación colectiva influye, como la relación entre el crecimiento salarial y la inflación, los niveles salariales de las nuevas contrataciones, y sobre todo, la respuesta de los salarios a las perturbaciones económicas.

Por este motivo, resulta de interés poder medir de algún modo la falta de respuesta de las diferencias salariales a las diferencias en la productividad de las empresas españolas. Como consecuencia de ello, estudiar el efecto que ese grado de rigidez genera en el empleo podría ayudarnos a encontrar una evidencia sobre la intensa destrucción de empleo que ha tenido lugar en los últimos años en España.

Así pues, en este artículo se pretende estudiar el efecto del sistema español de negociación colectiva sobre el crecimiento del empleo a través de una vía indirecta: cuantificando el grado de rigidez que provoca el mecanismo para fijar los salarios en España. El supuesto de partida es que la negociación colectiva, sobre todo a través de los convenios de ámbito superior, es la causa principal de la rigidez salarial del mercado laboral español. A partir de esta idea se propone la 
construcción de un indicador de rigidez del coste laboral. Posteriormente, se plantea un modelo que utiliza dicho índice como variable explicativa de las variaciones del empleo durante el periodo 1995-2013 para un conjunto de sectores de la economía española. Trataremos, en primer lugar, de explicar cómo se construye el índice en cuestión y a continuación procederemos al planteamiento y estimación del modelo propuesto.

Por tanto, el objetivo de este artículo es poner de manifiesto que un sistema de relaciones laborales basado en la negociación colectiva, sobre todo a través de los convenios sectoriales y provinciales, genera rigidez salarial, y, como vía de escape a dicha rigidez las empresas recurrirán a ajustar sus plantillas a través del empleo o a otro tipo de incentivos, como por ejemplo, la contratación temporal. Además, se trata de un enfoque que puede abrir nuevos caminos para futuras investigaciones; sobre todo a partir de lo que significa la medición del grado de rigidez salarial y la cuantificación de sus efectos sobre el crecimiento del empleo.

Vamos a estructurar el resto del trabajo del siguiente modo. En la sección segunda se comentarán algunas cuestiones relativas al sistema español de negociación colectiva y a su efecto en la rigidez del coste laboral. Posteriormente se procederá a construir el índice de rigidez del coste laboral. En el capítulo tercero se presentará el modelo a estimar. En el cuarto apartado se mencionarán los datos empleados y la metodología utilizada para estimar dicho modelo. En el apartado quinto se presentarán los principales resultados obtenidos tras la estimación y diversas pruebas de robustez del modelo y, por último, se presentarán las principales conclusiones en el apartado sexto.

\section{La rigidez del coste laboral en España}

El índice de rigidez salarial que se propone en este artículo se construye a partir de los datos de dispersión salarial. Para medir la dispersión de los salarios partimos de un hecho ampliamente reconocido: se dice que el sistema español de negociación colectiva es muy rígido porque impide que los salarios se adapten a las condiciones específicas de cada empresa; lo cual parece implicar que el sistema en cuestión impone unos salarios bastante uniformes ${ }^{3}$. Podemos pensar pues que el sistema

\footnotetext{
${ }^{3}$ Freeman (1980) ya comprobó que la presencia de sindicatos reduce la dispersión de los salarios porque, en el sector sindicalizado, la remuneración de las características del trabajador es más baja. Esto lo corroboran también Cardoso y Portugal (2005), que aseguran que un mayor poder sindical está asociado con remuneraciones menores a los
} 
de negociación colectiva hace que la dispersión salarial se comprima dentro de cada sector de la economía, aunque no en todos los sectores el efecto sea el mismo.

Todo esto parece verificarse, a un nivel muy intuitivo, a través del Gráfico 1, donde se compara la dispersión de la productividad (valor añadido por trabajador empleado) con la dispersión del coste laboral por trabajador para el conjunto de la industria española a través de una serie de años. En dicho gráfico no sólo se puede apreciar que la dispersión del coste laboral es mucho menor que la dispersión de la productividad; también se ve que esta última ha experimentado cambios sustanciales a lo largo del periodo 1995-2014, en tanto que la primera apenas ha variado 4 . Por otra parte, en el gráfico del anexo A.1 puede verse que el desglose por provincias muestra diferencias sustanciales en la dispersión de los costes laborales, aun tratándose de una dispersión muy inferior a la de la productividad, algo que también se evidenciará más adelante también a nivel sectorial a través del índice de rigidez que se presenta.

Uno de los factores que puede afectar a la distancia entre las dos dispersiones puede ser el sistema de negociación colectiva. Dentro de cada sector coexiste una pluralidad de empresas, unas mejor organizadas que otras, gracias a lo cual se generan rentas de mayor o menor cuantía. Normalmente, la negociación colectiva hace que las empresas compartan estas rentas con sus empleados. Aunque aquí habría que suponer un determinado tipo de negociación colectiva; en concreto, habría que suponer que cada empresa negocia por separado con sus propios trabajadores. Hablamos pues de convenios de empresa. Cuando los convenios se negocian a nivel sectorial, el escenario es completamente distinto. Sobre todo, en un escenario como el español, donde predominan los convenios sectoriales y provinciales. Aquí es muy probable que los intereses de los negociadores, sobre todo por el lado sindical, vayan más en la dirección de evitar desigualdades entre los trabajadores de un mismo sector que en sentido de adaptarse a la situación específica de cada empresa.

atributos del trabajador, tales como la educación, la antigüedad y la edad, lo que se debe al ánimo de los sindicatos de comprimir la dispersión salarial tratando de incrementar el nivel salarial global. Esta idea se podría justificar en base al modelo propuesto por Rodríguez Gutiérrez (2001). También en Canal y Rodríguez Gutiérrez (2004) se demuestra que las empresas con convenios colectivos de empresa muestran una dispersión salarial mayor que aquéllas cubiertas por convenios sectoriales.

${ }^{4}$ La uniformidad de la dispersión del coste laboral parece mantenerse aun modificando la escala del gráfico. 


\section{Gráfico 1: Dispersión de la productividad y dispersión del coste laboral.}

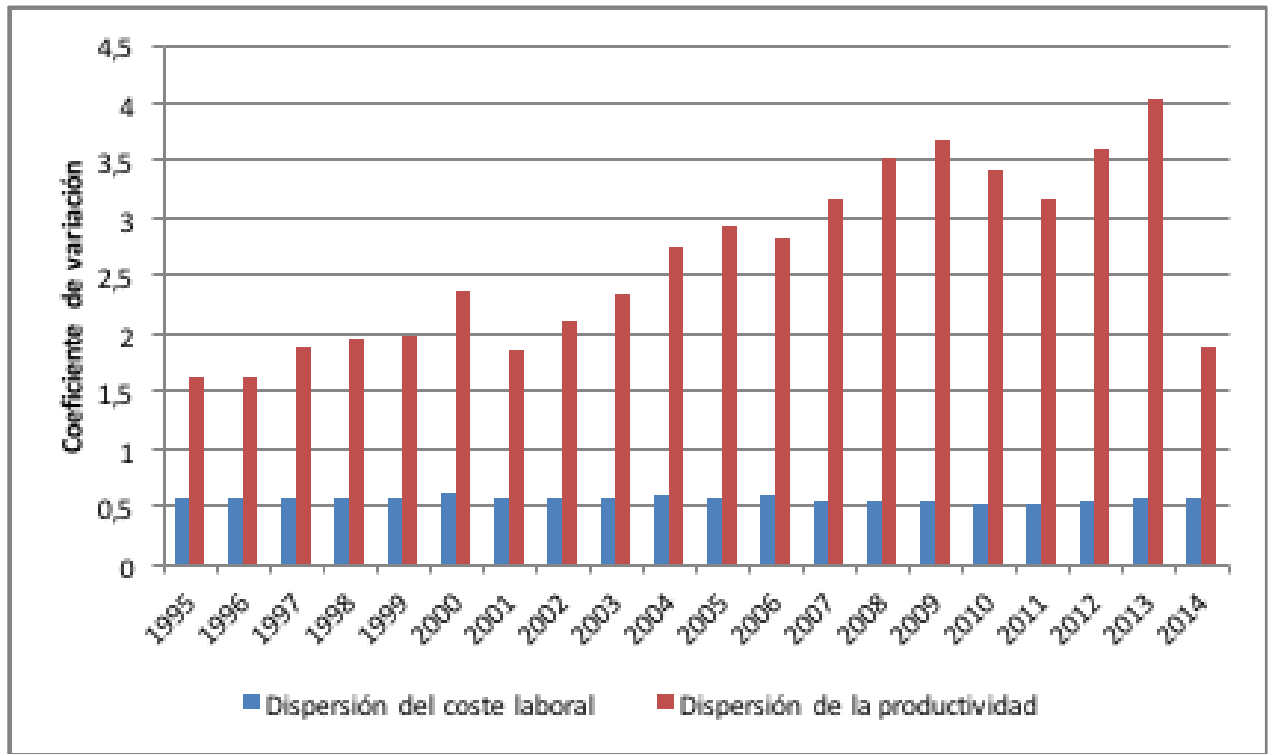

Fuente: elaboración propia a partir de Central de Balances.

Nota: la dispersión la hemos medido a través del coeficiente de variación. La productividad se ha medido con el valor medio de la producción por trabajador a precios corrientes.

La literatura ya ha documentado en diversas ocasiones que los países en que los salarios se negocian colectivamente en mayor medida también son aquellos que presentan mayores índices de rigideces salariales ${ }^{5}$. Por tanto, el sistema español de negociación colectiva hace que la dispersión del coste laboral no se corresponda con la dispersión de la productividad de las distintas ramas de la economía, tendiendo a uniformizar los costes laborales. Izquierdo et al. (2012) han utilizado datos de la Muestra Continua de Vidas Laborales para verificar que una mayor cobertura de convenios de empresa ${ }^{6}$ se asocia con una mayor dispersión de los salarios del sector correspondiente. Como en España la

\footnotetext{
5 Además, en estos países se ha observado también que los convenios colectivos han tendido a revisarse infrecuentemente, reflejando en momentos de crisis subidas salariales típicas de momentos expansivos, lo cual ha podido repercutir negativamente sobre el empleo. Para un detalle de estos efectos en España, conviene consultar el trabajo de Villanueva (2015).

${ }_{6}^{6}$ Para medir esta cobertura de convenios de empresa, los autores utilizan el porcentaje de trabajadores cubiertos por ese tipo de convenios.
} 
mayoría de los trabajadores están cubiertos por convenios de ámbito superior $^{7}$, esto impide que los salarios se adapten a las condiciones específicas de cada empresa. En las ramas donde priman los convenios colectivos de ámbito superior tienden a imponerse unos salarios bastante uniformes para todas las empresas con independencia de su productividad.

A partir de estas ideas podemos construir un índice, $R$, que llamaremos "índice de rigidez del coste laboral" del sector $i$ en el año $t$. Este índice mide el grado de adaptación de los costes laborales a las condiciones específicas de cada empresa y se define como sigue:

$$
R_{\mathrm{it}}=\frac{P_{\mathrm{it}}-D_{\mathrm{it}}}{P_{\mathrm{it}}}
$$

En esta expresión, $P_{i t}$ representa la dispersión de la productividad y $D_{i t}$ la dispersión de los salarios del sector $i$ en el año $t$. El índice en cuestión puede variar entre cero ${ }^{8}$ (rigidez mínima, cuando $P_{i t}=D_{i t}$ ) y uno (rigidez máxima, cuando la dispersión del coste laboral es nula, es decir, cuando $D_{i t}=0$ ). Cuanto mayor sea la distancia entre la dispersión de la productividad $\left(P_{i t}\right)^{9}$ y la dispersión del coste laboral $\left(D_{i t}\right)$, mayor será la rigidez del coste laboral. El índice se ha elaborado para ramas de actividad, con un nivel de desagregación de 2 dígitos según la CNAE de 2009 .

En el Cuadro 1 se presenta el promedio del índice de rigidez del coste laboral para los principales sectores de la economía, mientras que en el Apéndice (Tablas A.1 y A.2) se presenta dicho índice para un promedio del periodo 1995-2014 por rama de actividad a dos dígitos. Para

\footnotetext{
${ }^{7}$ En realidad en España el número de convenios de empresa es mayor que el número de convenios de ámbito superior (según el Registro de Convenios Colectivos en 2011 tan sólo un $31,5 \%$ de los convenios que se registraron eran de ámbito superior al de empresa). Sin embargo, la inmensa mayoría de los trabajadores españoles están cubiertos por el segundo tipo de convenio. Según la Encuesta de Estructura Salarial, el porcentaje de trabajadores cubierto por convenio de ámbito superior es del 80\%.

${ }^{8}$ En algunos casos especiales se ha obtenido un índice de rigidez menor de cero. En estos casos lo que ocurre es que la dispersión del coste laboral es ligeramente superior a la de la productividad. Dado que este hecho suele estar asociado a incrementos extraordinarios en las percepciones no salariales de alguna empresa de ese sector, hemos optado por ajustar tales valores a cero.

9 Durante el periodo 1995-2014, el valor de la dispersión de la productividad de los distintos sectores varía entre 0,5 y 35,3. El valor de la dispersión del coste laboral varía entre 0,2 y 4,5. Las empresas que tienen una productividad muy reducida se tienen en cuenta en el cálculo, y por ello, la dispersión de la productividad tiene valores tan elevados en algunos sectores y años.
} 
el conjunto del periodo, las actividades inmobiliarias, el comercio y la construcción parecen tener el índice de rigidez más alto. Esto puede deberse a que en estos sectores, la dispersión de la productividad es más amplia que la de los salarios, provocando diferencias mayores entre ambas y, por ello, un índice más alto. El incremento en la dispersión de la productividad también puede ser una de las razones que explique que, con la llegada de la última crisis económica, el índice de rigidez se incremente para la práctica mayoría de sectores en el periodo 2008-2014. Ya hemos observado antes que, en contraste con el incremento de la dispersión de la productividad, la dispersión de los salarios apenas se modifica con la crisis económica.

Cuadro 1: Promedio del índice de rigidez del coste laboral en los principales sectores.

\begin{tabular}{lcccc}
\hline Sectores & $\begin{array}{c}\mathbf{1 9 9 5}- \\
\mathbf{2 0 0 0}\end{array}$ & $\begin{array}{c}\mathbf{2 0 0 1 -} \\
\mathbf{2 0 0 7}\end{array}$ & $\begin{array}{c}\mathbf{2 0 0 8}- \\
\mathbf{2 0 1 4}\end{array}$ & $\begin{array}{c}\mathbf{1 9 9 5}- \\
\mathbf{2 0 1 4}\end{array}$ \\
\hline Industrias extractivas & 0,5650 & 0,5867 & 0,6394 & 0,5986 \\
Industria manufacturera & 0,6585 & 0,6605 & 0,7107 & 0,6775 \\
Suministro energía y gas & 0,5957 & 0,7133 & 0,7816 & 0,7019 \\
Gestión de residuos & 0,5547 & 0,6281 & 0,6989 & 0,6309 \\
Construcción & 0,7350 & 0,7878 & 0,8640 & 0,7986 \\
Comercio y reparación & 0,7193 & 0,7994 & 0,8492 & 0,7928 \\
Transporte y almacenamiento & 0,6296 & 0,6828 & 0,8117 & 0,7119 \\
Hostelería & 0,6285 & 0,7170 & 0,7913 & 0,7165 \\
Información y comunicaciones & 0,6297 & 0,7230 & 0,7800 & 0,7150 \\
A. inmobiliarias & 0,7227 & 0,8412 & 0,8643 & 0,8137 \\
A. profesionales & 0,5924 & 0,7094 & 0,7397 & 0,6849 \\
A. administrativas & 0,7375 & 0,8181 & 0,7763 & 0,7793 \\
Educación & 0,5646 & 0,7288 & 0,7894 & 0,7008 \\
A. sanitarias y ss. sociales & 0,4929 & 0,6568 & 0,6887 & 0,6188 \\
A. artísticas y recreativas & 0,4519 & 0,6718 & 0,7247 & 0,6243 \\
Otros servicios & 0,5246 & 0,6390 & 0,6467 & 0,6073 \\
TOTAL & 0,6127 & 0,7102 & 0,7598 & 0,6983 \\
\hline
\end{tabular}

Fuente: elaboración propia a partir de la Central de Balances.

Nota: este índice se ha elaborado utilizando la dispersión de la producción mediante la variable relativa al valor de la producción de cada empresa, aunque se ha construido un índice de rigidez similar con la dispersión del valor añadido bruto exento de otros conceptos (amortizaciones, subvenciones...), obteniéndose resultados muy similares. 


\section{Modelo empírico}

Una vez definido el índice de rigidez del coste laboral, podemos proceder a la especificación de un nuevo modelo que explique el crecimiento del empleo en función del efecto que esta rigidez genera en el mercado de trabajo. Para analizar el efecto de la rigidez del coste laboral sobre el crecimiento del empleo vamos a estimar un modelo en el cual haremos depender la tasa de variación del empleo, $\dot{L}$, de dos variables principales: la tasa de variación de la producción de la empresa, $\dot{Y}$, y el coeficiente de rigidez del coste laboral, $R$, además de una serie de controles del sector económico y efectos fijos de empresa, $\mathbf{S}^{\prime}$. Vamos a estimar este modelo en dos versiones alternativas, en función de la muestra de empresas con la que estemos trabajando: un panel no balanceado con todas las empresas de la muestra durante el periodo 1995-2014 (apartado 5.1), y un panel balanceado con las empresas que perduran en la muestra durante el periodo 1995-2014 (apartado 5.2).

La versión básica del modelo (ESTIMACIÓN 1), donde $i$ hace referencia a la empresa y $t$ al año del periodo 1995-2013, será pues:

$$
\dot{L}_{i t}=\gamma_{0}+\gamma_{1} \cdot \dot{Y}_{i t}+\gamma_{2} \cdot R_{i t}+\vec{\gamma}_{3} \cdot \vec{S}+v_{i t}
$$

Cabe pensar que las oscilaciones en la producción afectan de forma positiva al crecimiento del empleo. En tanto en cuanto la producción de los distintos sectores crezca, éstos demandarán más mano de obra para abastecer los incrementos de output.

A su vez, existe un factor institucional que afecta al crecimiento del empleo. La configuración institucional del mercado de trabajo influye sobre las decisiones relativas a la plantilla, tanto en relación con el coste laboral de las empresas como en relación con sus decisiones de ajuste de empleo. En España, el sistema de relaciones laborales afecta de forma sustancial al funcionamiento del mercado de trabajo, ya que establece una serie de limitaciones al despido y a la contratación. También afecta a la fijación de salarios estableciendo, por medio de convenios colectivos, unas tarifas salariales y unas condiciones mínimas a partir de las cuales las empresas pueden llevar a cabo sus decisiones. Por tanto, la rigidez del coste laboral influirá sobre la tasa de crecimiento del empleo de una manera directa, en la medida en que la respuesta ante las variaciones cíclicas de la economía dependa de dicha rigidez. En función del grado de rigidez de cada sector, las empresas podrán modificar los salarios de sus trabajadores o bien recurrirán a ajustar sus plantillas. 
La segunda versión (ESTIMACIÓN 2) incluye una dummy representativa del cambio estructural, $E$. Se supone que el cambio estructural afecta de manera distinta a cada empresa, dependiendo del grado de rigidez del coste laboral. Este hecho se podría reflejar incluyendo una dummy que llamaremos $E$ interaccionada por el índice de rigidez ${ }^{10}$, definida como sigue:

$$
\mathrm{E}=\left\{\begin{array}{l}
0, \text { si } t=1995, \ldots, 2007 \\
1, \text { si } t=2008, \ldots, 2014
\end{array}\right.
$$

Esta nueva versión del modelo quedaría reflejada en la siguiente ecuación:

$$
\dot{L}_{i t}=\gamma_{0}+\gamma_{1} \cdot \dot{Y}_{i t}+\gamma_{2} \cdot R_{i t}+\vec{\gamma}_{3} \cdot \vec{S}+\gamma_{4} \cdot R_{i t} \cdot E+v_{i t}
$$

\section{Datos y metodología}

Para realizar este trabajo se han utilizado datos de la Central de Balances del Banco de España. La Central de Balances contiene información contable y financiera a partir de las cuentas anuales que las empresas no financieras depositan en el Registro Mercantil. Debido a la peculiaridad de esta base de datos, conviene considerar que la representatividad de las empresas presentes en ella no es uniforme según las características de las empresas, y sobre todo, puede ser inferior en el caso de empresas pequeñas o con dificultades. Para controlar este hecho, se ha introducido una prueba de robustez utilizando datos procedentes de otras bases de datos, tal y como se presenta en el Apéndice (Tabla A.3), que aparece explicado más adelante.

Para seleccionar las empresas utilizadas en la estimación, hemos eliminado aquellas con valores por encima y por debajo del 1\% de su coste laboral y de su producción. El número de empresas que utilizaremos en la estimación es de 1030951 empresas.

\footnotetext{
${ }^{10}$ La interacción pretende recoger el efecto que ha podido tener la crisis en la propia rigidez. Esto es debido, principalmente, al incremento en la dispersión de la productividad como consecuencia de una época recesiva. Además, este hecho también ha afectado, en segundo término, al indicador de rigidez, ya que como hemos mencionado anteriormente, han existido sectores menos proclives o más lentos en el ajuste de sus salarios como consecuencia de la negociación colectiva, lo que ha hecho que la diferencia entre la dispersión salarial y la dispersión de la productividad haya podido incrementarse notablemente durante la crisis.
} 
A su vez, hemos asignado el índice de rigidez a cada una de las empresas a través del sector al que pertenecen, a dos dígitos, siguiendo la CNAE 2009.

En el Cuadro 2 se muestran los principales estadísticos de la base de datos.

Cuadro 2: Principales estadísticos descriptivos.

\begin{tabular}{|c|c|c|c|c|c|}
\hline Variable & Obs. & Media & $\begin{array}{l}\text { Desv. } \\
\text { est. }\end{array}$ & Mín. & Máx. \\
\hline Producción & 9069166 & 1884,77 & 60702,97 & 0 & $\begin{array}{c}2,34 \mathrm{E}+0 \\
7\end{array}$ \\
\hline Empleo & 9072219 & 10,3631 & 216,6744 & 0 & 71333 \\
\hline Empleo (trabajadores indefinidos) & 9072219 & 7,6696 & 176,2375 & 0 & 70868 \\
\hline Coste laboral por trabajador & 9072177 & 290,989 & 6842,335 & 0 & 2674017 \\
\hline Dispersión del coste laboral & 9072066 & 0,5986 & 0,2783 & 0,2340 & 4,4698 \\
\hline Dispersión de la producción & 9072066 & 4,1658 & 3,5061 & 0,5975 & 35,2836 \\
\hline Índice de rigidez del coste laboral & 9072066 & 0,7892 & 0,1250 & 0 & 0,9791 \\
\hline Número de ocupados (EPA) & 7797657 & 607,4461 & 555,8169 & 1,1 & 2015,5 \\
\hline Tasa de crecimiento del empleo & 5772519 & 0,1699 & 12,7690 & -1 & 29069,59 \\
\hline $\begin{array}{l}\text { Tasa de crecimiento del empleo } \\
\text { (trabajadores indefinidos) }\end{array}$ & 5470222 & 0,1691 & 7,4822 & -1 & 15404,88 \\
\hline $\begin{array}{l}\text { Tasa de crecimiento del coste } \\
\text { laboral por trabajador }\end{array}$ & 5782644 & 0,2487 & 10,2933 & -1 & 18004,49 \\
\hline $\begin{array}{l}\text { Tasa de crecimiento de la } \\
\text { producción }\end{array}$ & 6601393 & 1,4058 & 208,4609 & -1 & 235388 \\
\hline $\begin{array}{l}\text { Tasa de crecimiento del número de } \\
\text { ocupados (EPA) }\end{array}$ & 5949922 & 0,0971 & 2,4507 & $-0,9994$ & 1491,963 \\
\hline
\end{tabular}

Fuente: elaboración propia a partir de la Central de Balances y Encuesta de Población Activa.

Para construir la tasa de crecimiento del empleo $(\dot{L})$ se ha utilizado la variable que mide el número de trabajadores de cada empresa en cada año. La tasa de crecimiento de la producción $(\dot{Y})$ se ha construido a partir del valor añadido de la empresa. En cuanto al índice de rigidez, $R$, hay que decir que se construye tal y como hemos detallado en secciones previas.

También conviene aclarar que hemos utilizado diversos controles alternativos de provincia o rama de actividad en las distintas especificaciones, así como efectos fijos a nivel de empresa en todas ellas (lo que denominábamos como $S$ ).

También de forma alternativa, en lugar de utilizar el número de trabajadores total para construir la tasa de crecimiento del empleo, 
hemos utilizado únicamente el número de trabajadores fijos de las empresas supervivientes. De este modo, nos centramos en el colectivo de trabajadores con mayor cobertura de la negociación colectiva, lo cual puede tener un efecto notable en la determinación de sus condiciones laborales y de sus salarios, así como aportarnos información relevante sobre si existen diferencias en los resultados para este colectivo de trabajadores. Los resultados de esta estimación se muestran en el apartado 5.3. Por último, para tratar de comprobar si el indicador de rigidez del coste laboral es consistente y ha podido tener algún efecto también sobre la flexibilidad interna de las empresas, se ha estimado el mismo modelo pero empleado datos del tiempo trabajado, en lugar del total de empleo, a partir de la Encuesta de Población Activa. Asimismo, en esta estimación se ha incluido como prueba de robustez los datos de rigidez construidos a partir de la base de datos AMADEUS y los datos de productividad a partir del Valor Añadido Bruto a nivel de sector suministrados por la Contabilidad Nacional.

\section{Resultados}

\subsection{El impacto de la rigidez del coste laboral sobre el crecimiento del empleo}

Los resultados de la estimación del modelo se presentan en el Cuadro 3. En la estimación del modelo básico se comprueba, en primer lugar, que todas las variables explicativas son significativas y presentan los signos esperados: el crecimiento de la producción afecta de forma positiva al crecimiento del empleo sectorial, y el índice de rigidez tiene un efecto negativo sobre el crecimiento del empleo. Esto último querría decir que al aumentar la rigidez de un sector, el crecimiento del empleo sería menor. Concretamente, si el índice aumentara un 10\% el empleo se desaceleraría aproximadamente un 18\%, dependiendo de la estimación. Se confirma pues la hipótesis de partida, según la cual una mayor rigidez del coste laboral genera un crecimiento del empleo más lento. De hecho, si comparamos un sector con rigidez alta (cercana a 1) con otro de rigidez baja (cercano a 0), en el sector más rígido el empleo se desaceleró un 18\% más.

Los resultados que se extraen de la segunda especificación nos confirman otra hipótesis inicial. El cambio estructural de 2008 tiene una incidencia más negativa a medida que la rigidez es más alta, es decir, dicho cambio ha resultado más significativo y más negativo en los 
sectores más rígidos. Ello supone que, en estos sectores, su empleo ha decrecido más desde 2008.

Cuadro 3: Resultados de la estimación del crecimiento del empleo.

\begin{tabular}{|c|c|c|c|c|}
\hline \multicolumn{5}{|c|}{ VARIABLE DEPENDIENTE: Lit } \\
\hline \multirow[t]{2}{*}{ VARIABLES } & \multicolumn{2}{|c|}{ ESTIMACIÓN 1} & \multicolumn{2}{|c|}{ ESTIMACIÓN 2} \\
\hline & (a) & (b) & (a) & (b) \\
\hline$\dot{Y}_{i t}$ & $0,000614^{* * *}$ & $0,000614^{* * *}$ & $0,000613^{* * *}$ & $0,000613^{* * *}$ \\
\hline$R^{i t}$ & $-0,184 * * *$ & $-0,179 * * *$ & $0,190 * * *$ & $0,187^{* * *}$ \\
\hline$R^{*} E$ & & & $-0,304^{* * *}$ & $-0,305^{* * *}$ \\
\hline Constante & 0,253 & $1,697 * *$ & 0,192 & $1,500 * *$ \\
\hline Ramas actividad & Sí & NO & SÍ & NO \\
\hline Provincias & NO & SÍ & NO & SÍ \\
\hline $\mathrm{N}$ & 5726783 & 5726783 & 5726783 & 5726783 \\
\hline $\mathrm{R}^{2}$ & 0,001 & 0,001 & 0,002 & 0,002 \\
\hline
\end{tabular}

Fuente: elaboración propia a partir de la Central de Balances.

Nota: $\left.{ }^{*}\right)$ representa significatividad al $10 \%,(* *)$ significatividad al $5 \%,(* * *)$ significatividad al $1 \%$.

Por tanto, podemos concluir que un incremento de la rigidez ralentiza el crecimiento del empleo en las épocas de auge del ciclo económico y provoca un decrecimiento a un ritmo mayor en épocas recesivas; es decir, cuanto mayor sea la rigidez de un sector (la dificultad de adaptación de sus salarios a los cambios en la productividad), más lento será el crecimiento del empleo en los periodos expansivos, y más decaerá en los periodos de crisis. Esto se explica porque las empresas no pueden ajustar tan fácilmente su situación interna a las condiciones del mercado, y por ello son más reticentes a contratar mano de obra y son más propensas a destruirlo en las épocas recesivas.

\subsection{La rigidez del coste laboral y el crecimiento de empleo en las empresas supervivientes}

Para comprobar hasta qué punto han podido existir diferencias en el ajuste de las empresas durante la crisis económica, hemos realizado el mismo experimento estimando únicamente una muestra de empresas 
que están presentes en la muestra todos los años del periodo 1995-2014. El número de empresas que consideramos es de 17592 empresas. Los resultados de las estimaciones de los modelos anteriormente descritos se presentan en este caso en el Cuadro 4.

\section{Cuadro 4: Resultados de la estimación del crecimiento del} empleo en las empresas supervivientes.

\begin{tabular}{|c|c|c|c|c|}
\hline \multicolumn{5}{|c|}{ VARIABLE DEPENDIENTE: $\mathrm{L}_{\mathrm{it}}$} \\
\hline \multirow[t]{2}{*}{ VARIABLES } & \multicolumn{2}{|c|}{ ESTIMACIÓN 1} & \multicolumn{2}{|c|}{ ESTIMACIÓN 2} \\
\hline & (a) & (b) & (a) & (b) \\
\hline$\dot{Y}$ & $3,10 \mathrm{e}-05$ & $3,11 \mathrm{e}-05$ & 0,000032 & 0,000033 \\
\hline$R^{i t}$ & $-0,318^{* * *}$ & $-0,305^{* * *}$ & $-0,0959 * * *$ & $-0,0881^{* * *}$ \\
\hline$R^{*} E$ & & & $-0,1163^{* * *}$ & $-0,1181^{* * *}$ \\
\hline Constante & 0,200 & 0,309 & 0,161 & 0,148 \\
\hline Ramas de actividad & SÍ & NO & SÍ & NO \\
\hline Provincias & NO & SÍ & NO & SÍ \\
\hline $\mathrm{N}$ & 215207 & 215207 & 215207 & 215207 \\
\hline $\mathrm{R}^{2}$ & 0,002 & 0,003 & 0,001 & 0,001 \\
\hline
\end{tabular}

Fuente: elaboración propia a partir de la Central de Balances.

Nota: $\left(^{*}\right)$ representa significatividad al $10 \%,(* *)$ significatividad al $5 \%,(* * *)$ significatividad al $1 \%$.

Se observa que los resultados del apartado anterior se confirman al emplear solamente aquellas empresas presentes en la muestra durante todo el periodo. Además, podemos ver que el impacto negativo de la rigidez sobre el crecimiento del empleo es incluso mayor en estas empresas: si la rigidez se incrementa un 10\%, el empleo se desacelera en un $30 \%$. Además, en el caso de la interacción de la dummy que refleja la crisis económica, se observa que desde 2008 el efecto de la rigidez sobre el crecimiento del empleo es aún mayor. Esto quiere decir que las empresas que operan en sectores con mayor rigidez tienden a desacelerar su empleo en mayor medida durante la crisis económica. Este impacto es mayor que en cuando consideramos la muestra total de empresas. Resulta remarcable comprobar cómo precisamente las empresas que han sobrevivido en la muestra durante las épocas recesivas son las que 
muestran una mayor sensibilidad del crecimiento del empleo a la rigidez del sector.

\subsection{La rigidez del coste laboral y el crecimiento del empleo indefinido}

Los resultados de las estimaciones para el empleo indefinido de las empresas supervivientes de la muestra se presentan en el Cuadro 5. En este caso, el número de empresas que hemos utilizado en la muestra es de 17464 empresas.

Cuadro 5: Resultados de la estimación del crecimiento del empleo indefinido en las empresas supervivientes.

\begin{tabular}{lcccc}
\hline \multicolumn{2}{l}{ VARIABLE DEPENDIENTE: $\mathrm{L}_{\mathrm{it}}$} & \multicolumn{2}{c}{ ESTIMACIÓN 2} \\
\cline { 2 - 5 } VARIABLES & \multicolumn{2}{c}{ ESTIMACIÓN 1} & (a) & (b) \\
$\dot{Y}_{i t}$ & $2,07 \mathrm{e}-05$ & $2,21 \mathrm{e}-05$ & $2,24 \mathrm{e}-05$ & $2,38 \mathrm{e}-05$ \\
$R^{*} E$ & $-0,383^{* * *}$ & $-0,364^{* * *}$ & $-0,107^{* * *}$ & $-0,0956^{* * *}$ \\
$R^{*} E$ & & & $-0,144^{* * *}$ & $-0,145^{* * *}$ \\
Constante & 0,189 & 0,356 & 0,141 & 0,157 \\
Ramas de actividad & SÍ & NO & SÍ & NO \\
Provincias & NO & SÍ & NO & SÍ \\
N & 209485 & 209485 & 209485 & 209485 \\
$R^{2}$ & 0,002 & 0,002 & 0,004 & 0,004 \\
\hline
\end{tabular}

Fuente: elaboración propia a partir de la Central de Balances.

Nota: (*) representa significatividad al 10\%, $\left(^{* *}\right)$ significatividad al 5\%, (***) significatividad al $1 \%$.

Se puede comprobar cómo el efecto negativo de la rigidez sobre el crecimiento del empleo indefinido no sólo se mantiene sino que se hace aún más intenso. En este caso, un incremento del 10\% de la rigidez ocasionaría una desaceleración del empleo de entre un $35 \%$ y un $39 \%$, dependiendo del tipo de especificación y controles que estemos considerando. Si observamos lo que ocurre cuando introducimos la interacción con la crisis económica, este efecto negativo de la rigidez sobre el crecimiento del empleo se intensifica desde 2008. Además, el 
coeficiente de la interacción es algo mayor en el caso de considerar el empleo indefinido que cuando considerábamos el empleo total de las empresas. Esto quiere decir que en los colectivos con mayor cobertura de la negociación colectiva, pese a las mayores restricciones para el despido, el crecimiento del empleo en estas empresas se ve afectado especialmente en caso de producirse una época recesiva.

\section{Conclusiones}

En este artículo se ha tratado de analizar los efectos que la rigidez del coste laboral ha tenido en el crecimiento del empleo español durante los últimos años.

Se ha partido de la construcción de un indicador de rigidez del coste laboral, que refleja la distancia entre la dispersión del coste laboral y la dispersión de la productividad, para tratar de explicar el crecimiento del empleo a nivel sectorial. El principal resultado obtenido indica que la rigidez tiene un impacto negativo sobre el crecimiento del empleo. La rigidez del coste laboral ralentiza el crecimiento del empleo en las fases de alza y acelera su caída en las fases de depresión. Es probable que este impedimento para ajustar el empleo a la producción en los sectores con mayor rigidez del coste laboral provoque otras consecuencias, como el hecho de que tengan mayores tasas de temporalidad. Además, se observa que este impacto negativo de la rigidez sobre el crecimiento del empleo es aún mayor en las empresas que perduran en la muestra. Y también hemos podido comprobar cómo el efecto es también tanto más importante cuando consideramos únicamente el empleo indefinido de estas empresas, que es aquél con mayores niveles de cobertura de la negociación colectiva y el cuál es más difícil ajustar en caso de que las empresas lo requieran. Por tanto, cuanto mayor es la rigidez, más perjudicado se ve el crecimiento del empleo en este tipo de empresas.

En definitiva, el sistema español de negociación colectiva parece ser excesivamente rígido. Y la excesiva rigidez tiene consecuencias en la evolución del empleo: al tener mayores dificultades para ajustar el empleo a las variaciones de la producción y del coste laboral, el empleo es menos sensible a las épocas de expansión pero se destruye con mayor intensidad en las épocas recesivas. Introducir elementos de flexibilidad en el mercado de trabajo español sin duda ayudaría a las empresas a ajustarse mejor a los ciclos y eso a la larga tendría una repercusión positiva sobre el empleo 


\section{Bibliografía}

Bande, R.; Fernández, M. y Montuenga, V. M. (2007). "Regional Disparities in the Unemployment Rate: The Role of the Wage-setting Mechanism in Spain, 1987-92", Regional Studies, 41 (2), 235-251.

Bande, R.; Fernández, M. y Montuenga, V. M. (2010). "Desequilibrios territoriales en el mercado laboral español y estructura de la negociación colectiva”, Papeles de Economía Española, 124, 193-213.

Bentolila, S. y Jimeno, J. F. (2002). "La reforma de la negociación colectiva en España", Fundación de Estudios de Economía Aplicada (FEDEA), Documento de Trabajo 2002-03.

Bentolila, S.; Izquierdo, M. y Jimeno, J. F. (2010). "Negociación colectiva: la gran reforma pendiente", Papeles de Economía Española, 124, 176 194.

Canal Domínguez, J. F. y Rodríguez Gutiérrez, C. (2004). "Collective bargaining and within-firm wage dispersion in Spain", British Journal of Industrial Relations, 42 (3), 481-506.

Cardoso, A. y Portugal, P. (2005). "Contractual Wages and the Wage Cushion under Different Bargaining Settings", Journal of Labour Economics, 23(4), 875-902.

Freeman, R. B. (1980). "Unionism and the Dispersion of Wages", Industrial and Labor Relations Review, 34(1), 3-23.

Izquierdo, M.; Jimeno, J. F. y Thomas, C. (2012). "La relación entre el grado de centralización de la negociación colectiva y la tasa de desempleo: nuevos resultados", Boletín Económico del Banco de España, febrero de 2012, 59-66.

Lorences, J.; Fernández, V. y Rodríguez, C. (1995). "Diferencias interterritoriales de salarios y negociación colectiva en España", Investigaciones Económicas, 19 (2), mayo 1995, 309-324. 
Palacio, J. I. y Simón, H. (2004). "Dispersión salarial entre establecimientos y desigualdad salarial", Revista de Economía Aplicada, 12(36), 47-81.

Pérez Infante, J. I. (2003). "La estructura de la negociación colectiva y los salarios en España", Revista del Ministerio de Trabajo y Asuntos Sociales, 46, 41-97.

Rodríguez Gutiérrez, C. (2001). "Wage dispersion within firms and collective bargaining in Spain”, Economics Letters, 72(3), 381-386.

Villanueva, J. (2015). "Negociación colectiva, rigideces salariales y empleo: un análisis con datos microeconómicos", Boletín Económico del Banco de España, abril de 2015, 41-48. 


\section{Apéndice}

Tabla A1: Índice de rigidez medio del periodo 1995-2014 por ramas de actividad (CNAE 2009).

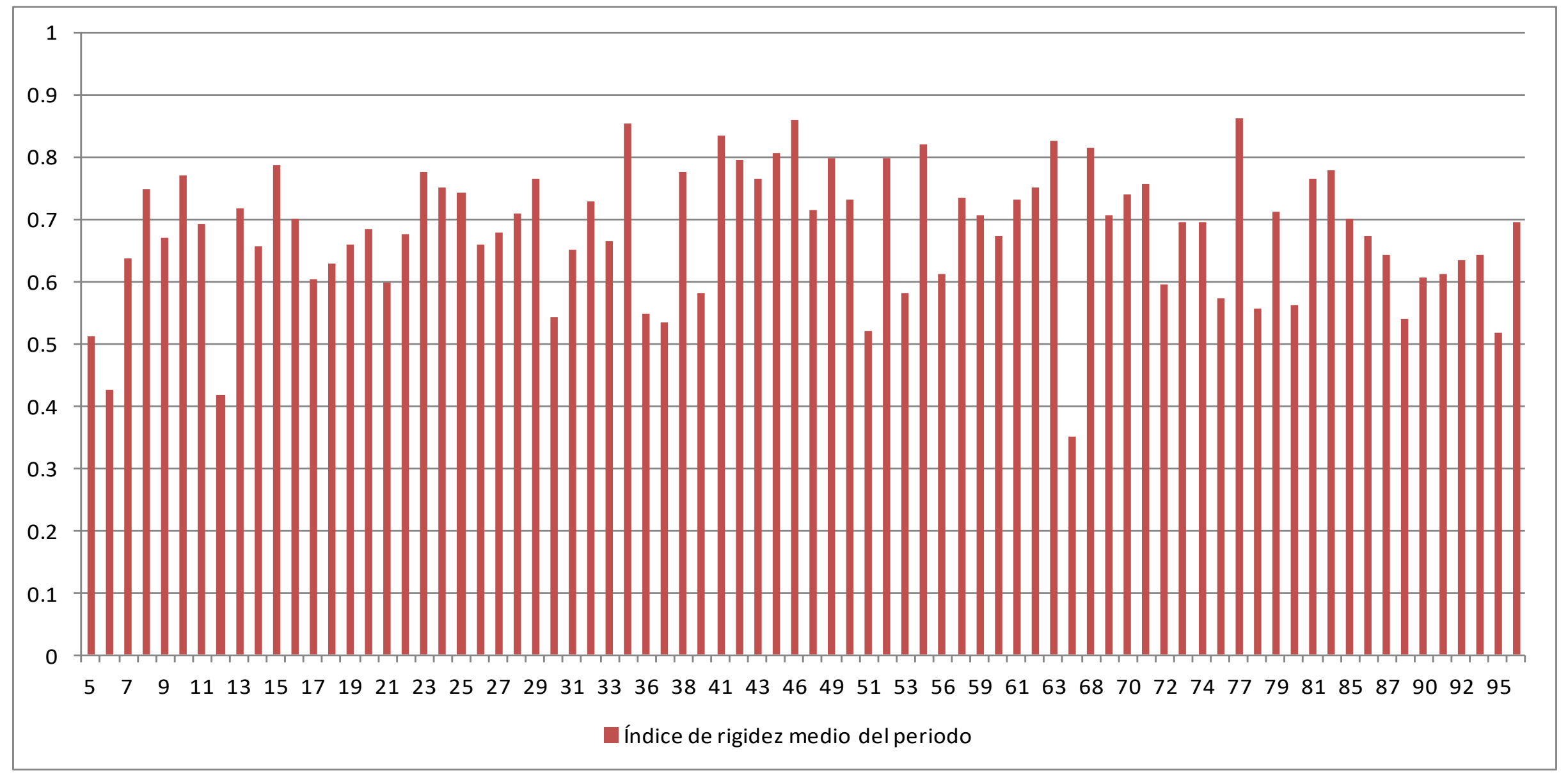

Fuente: elaboración propia a partir de la Central de Balances. 
Tabla A2: Índice de rigidez medio del periodo 1995-2014 por ramas de actividad.

\begin{tabular}{|c|c|}
\hline Rama de actividad (CNAE 2009-2 dígitos) & Indice de rigidez \\
\hline 5 & 0,5120512 \\
\hline 6 & 0,425569 \\
\hline 7 & 0,6363708 \\
\hline 8 & 0,747365 \\
\hline 9 & 0,6718006 \\
\hline 10 & 0,7714077 \\
\hline 11 & 0,6918182 \\
\hline 12 & 0,4183002 \\
\hline 13 & 0,7179855 \\
\hline 14 & 0,6556857 \\
\hline 15 & 0,7873521 \\
\hline 16 & 0,7017559 \\
\hline 17 & 0,6046473 \\
\hline 18 & 0,6280771 \\
\hline 19 & 0,6581099 \\
\hline 20 & 0,6838576 \\
\hline 21 & 0,5991982 \\
\hline 22 & 0,6747224 \\
\hline 23 & 0,7750291 \\
\hline 24 & 0,7513281 \\
\hline 25 & 0,74226 \\
\hline 26 & 0,6603312 \\
\hline 27 & 0,6775373 \\
\hline 28 & 0,7089649 \\
\hline 29 & 0,7640908 \\
\hline 30 & 0,5419276 \\
\hline 31 & 0,6510845 \\
\hline 32 & 0,7299894 \\
\hline 33 & 0,6644651 \\
\hline 35 & 0,8545154 \\
\hline 36 & 0,5493698 \\
\hline 37 & 0,5338436 \\
\hline 38 & 0,7767146 \\
\hline 39 & 0,582036 \\
\hline 41 & 0,8354578 \\
\hline 42 & 0,7963141 \\
\hline 43 & 0,7640715 \\
\hline 45 & 0,806198 \\
\hline 46 & 0,8587297 \\
\hline 47 & 0,7135262 \\
\hline 49 & 0,7979107 \\
\hline 50 & 0,7303351 \\
\hline 51 & 0,5206019 \\
\hline
\end{tabular}

(C) Revista de Economía Laboral 
154 Martínez Matute / Revista de Economía Laboral 13(2) (2016), 135-155

\begin{tabular}{|c|c|}
\hline 52 & 0,7988893 \\
\hline 53 & 0,582015 \\
\hline 55 & 0,8200837 \\
\hline 56 & 0,6128557 \\
\hline 58 & 0,7351484 \\
\hline 59 & 0,7062317 \\
\hline 60 & 0,6734384 \\
\hline 61 & 0,7309302 \\
\hline 62 & 0,7515168 \\
\hline 63 & 0,8256489 \\
\hline 64 & 0,3522904 \\
\hline 68 & 0,8136912 \\
\hline 69 & 0,7064968 \\
\hline 70 & 0,7392931 \\
\hline 71 & 0,7566592 \\
\hline 72 & 0,594661 \\
\hline 73 & 0,6957784 \\
\hline 74 & 0,6951807 \\
\hline 75 & 0,5722907 \\
\hline 77 & 0,8609151 \\
\hline 78 & 0,557195 \\
\hline 79 & 0,7122374 \\
\hline 80 & 0,5624031 \\
\hline 81 & 0,7656655 \\
\hline 82 & 0,7792997 \\
\hline 85 & 0,7007858 \\
\hline 86 & 0,6733936 \\
\hline 87 & 0,6434731 \\
\hline 88 & 0,5395728 \\
\hline 90 & 0,6073994 \\
\hline 91 & 0,6133602 \\
\hline 92 & 0,6345608 \\
\hline 93 & 0,6420375 \\
\hline 95 & 0,5178667 \\
\hline 96 & 0,6968204 \\
\hline
\end{tabular}

Fuente: elaboración propia a partir de la Central de Balances. 
Tabla A3: Resultados de la estimación para sectores con variables alternativas.

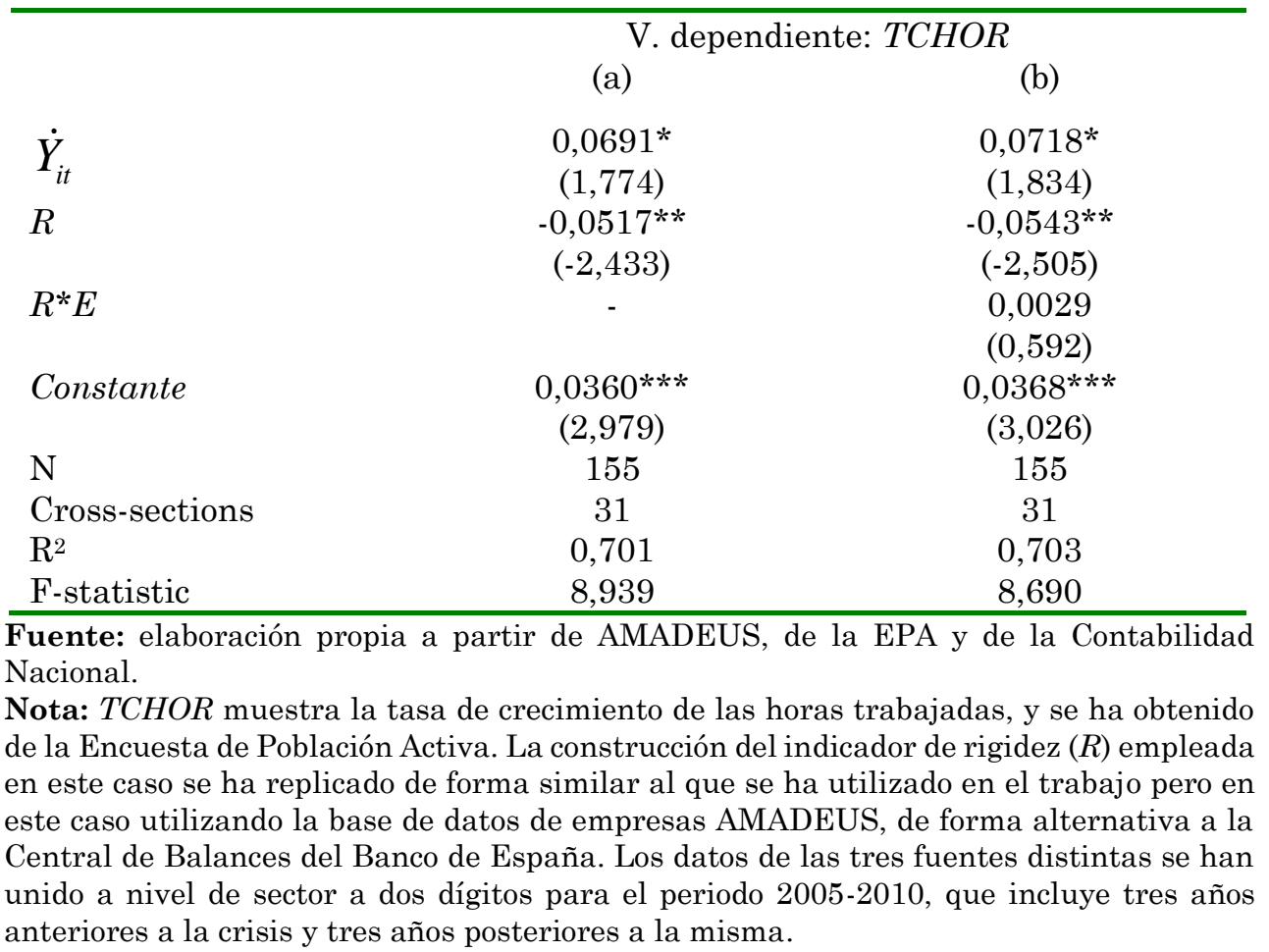

